\title{
Response to Soil Acidity of Common Bean Genotypes (Phaseolus vulgaris L.) Under Field Conditions at Nedjo, Western Ethiopia
}

\author{
Hirpa Legesse ${ }^{1}$, Nigussie-Dechassa $\mathbf{R}^{2}$, Setegn Gebeyehu ${ }^{3}$, Geremew Bultosa ${ }^{4}$ and \\ Firew Mekbib ${ }^{2}$
}
${ }^{1}$ Collage of Agriculture and Natural Resource, Post Box No: 395, Wollega University, Nekemte, Ethiopia
${ }^{2}$ Department of Plant Sciences, Haramaya University, Post Box No: 138, Dire Dawa, Ethiopia
${ }^{3}$ OA HARO, Post Box No: 25779, Addis Ababa, Ethiopia
${ }^{4}$ Department of Food Science and Technology, Botswana College of Agriculture, Gaborone

\begin{abstract}
Soil acidity has become a serious threat to crop production in most highlands of Ethiopia in general and in the western part of the country in particular. A field experiment was conducted to evaluate soil acidity tolerant on 25 genotypes of common bean (Phaseolus vulgaris L.) on lime treated and untreated acid soils. The genotypes were evaluated based on morpho-agronomic parameters. Soil acidity had an effect on maturity, growth and yield of the tested genotypes. High significant $(P<0.01)$ differences were observed among genotypes for days to maturity, leaf area index, plant height, number of pods per plant, pod length, hundred seed weight, grain yield, pod harvest index and harvest index in lime treated and untreated soils. Considerable variability exists among the genotypes tested based on the growth, and yield components measured for soil acidity tolerance. The genotypes gave higher yield and yield components when grown in lime treated soil than lime untreated with average yield reduction of $26 \%$ due to soil acidity effect. Relative grain yield was calculated as the ratio of grain yield in lime untreated to lime treated soil also showed higher significant differences among the tested genotypes. Genotypes such as Dimtu, new BILFA 58, Beshbesh, SER176, new BILFA51 and new BILFA 61 gave higher absolute and relative yield, whereas Dinknesh, Chore, Nasser and new BILFA 60 gave lower absolute grain and relative yield. Moreover, the great variability of 25 common bean genotypes exhibited a good potential to screening large germplasm of common bean for soil acidity tolerance and develop a cultivar that are tolerant to soil acidity in the country.
\end{abstract}

\section{Article Information Article History: \\ Received : 01-07-2013 \\ Revised : 25-08-2013 \\ Accepted : 30-08-2013}

\section{Keywords:}

Common bean

Genotypes

Lime

Soil acidity

Relative yield

${ }^{*}$ Corresponding Author:

Hirpa Legesse

E-mail:

hirpa.leg@gmail.com

\section{INTRODUCTION}

Acid soils make up approximately $30 \%$ of the world's total land area and more than $50 \%$ of the world's potentially arable lands, particularly in the tropics and subtropics (Kochian et al., 2004). When the $\mathrm{pH}$ drops to below 5, aluminium (Al) is released into the soil solution and becomes the single most important factor limiting crop production on $67 \%$ of the total acid soil area in the World (Eswaran et al., 1997). The extent of soil acidity in Africa is difficult to quantify. Eswaran et al. (1997) estimated that $28.8 \%$ of the African continent has acid surface soils and $19.6 \%$ has sub soil acidity problems. Soil acidity has become a serious threat to crop production in most highlands of Ethiopia in general and in the western part of the country in particular. Currently, it is estimated that about $40 \%$ of the total arable land of Ethiopia is affected by soil acidity (Abdenna et al., 2007; Mesfin, 2007).

Common bean is currently produced on about $331,708.15$ hectares of land in Ethiopia; with a total production of $387,802.3$ tonnes with an average yield of 1.17 tonnes/ha (CSA, 2012). Common bean is generally less adapted to acid soil 
Hirpa Legesse et al.,

environments and improving $\mathrm{Al}$ resistance of common bean to reduce the dependence of smallscale farmers on lime and nutrient inputs is a major challenge (Rao, 2001). However, efforts to develop adapted genotypes indicate that there are genotypic differences in Al resistance in the bean germplasm (Rao, 2001; Rangel et al., 2005; Manrique et al., 2006).

To mitigate severe yield reductions, smallholder farmers in the western and eastern Wollega zones have been abandoning their land temporarily (fallowing) or even permanently in some areas. However, owing to the increasing population pressure, abandoning farmland temporarily or permanently has become an untenable option. Therefore, the farmers are now opting for managing soil fertility to sustain productivity. Nevertheless, for both logistics and economic reasons, it is often not practicable for resource-poor farmers to apply high rates of lime as well as mineral fertilizers. There is, therefore, a need to develop practicable alternatives. For these reasons, development of cultivars adapted to acid soil complexes is a promising alternative or supplement to liming and related agronomic practices. Hence, the selection of common bean genotypes/varieties adapted to acid soil conditions of western Ethiopia is necessary to ensure economic stability to many farmers who
Sci. Technol. Arts Res. J., July-Sep 2013, 2(3): 03-15

cannot afford application of liming material. Common bean varieties with the capacity to tolerate acidic soil conditions will also produce high yields in areas where liming is not feasible due to high acidity conditions in the subsoil. Therefore the aim of this study was to assess the differential response of common bean genotypes of different origin to soil acidity in terms of growth, yield, and yield related traits under field conditions.

\section{MATERIALS AND METHODS}

\section{Description of the Study Site}

The field study was conducted at Nedjo TVET Collage, which is located in western Wollega zone of the Oromia Regional State at the latitude of $9^{\circ} 5^{\prime} \mathrm{N}$ and longitude of $35^{\circ} 45^{\prime} \mathrm{E}$ in western Ethiopia. The site has an elevation of 1735 meters above sea level with a gently undulating slope (0-5\%). The rainfall distribution is mono-modal with the long rains occurring from April to October. The annual rainfall during the experimental period was 1386 $\mathrm{mm}$ with mean minimum and maximum temperatures of $12^{\circ} \mathrm{C}$ and $26^{\circ} \mathrm{C}$, respectively. The soils are acidic, well drained, deep, and reddish brown in colour. The physical and some chemical properties of the soil in the study area before sowing and after harvesting the crop for both lime treated and untreated soil are indicted in Table 1.

Table 1: Physical and Chemical properties of the soil for field experiment site at Nedjo, western Ethiopia.

\begin{tabular}{|c|c|c|c|c|}
\hline \multirow{3}{*}{ No. } & \multirow{3}{*}{ Soil Analysis } & \multicolumn{3}{|l|}{ Field (Nejo) } \\
\hline & & \multirow{2}{*}{ Before sowing } & \multicolumn{2}{|c|}{ After Harvest } \\
\hline & & & Unlimed & Limed \\
\hline \multirow[t]{4}{*}{1} & Texture & Silt loam & Loam & loam \\
\hline & Clay (\%) & & & \\
\hline & Sand $(\%)$ & 35 & & \\
\hline & Silt (\%) & 50 & & \\
\hline 2 & $\mathrm{pH}\left(\mathrm{H}_{2} \mathrm{O}\right)$ & 4.45 & 4.39 & 5.14 \\
\hline 3 & Organic matter (\%) & 5.03 & 4.96 & 5.75 \\
\hline 4 & Total N (\%) & 0.25 & 0.27 & 0.30 \\
\hline 5 & Available P(Brayll)(ppm) & 7.96 & 6.95 & 10.43 \\
\hline 6 & EC & 0.090 & 0.123 & 0.11 \\
\hline \multirow[t]{6}{*}{7} & Cations (meq/100 g soil) & & & \\
\hline & $\mathrm{Ca}$ & 1.54 & 1.75 & 5.0 \\
\hline & $\mathrm{Mg}$ & 2.26 & 3.10 & 2.50 \\
\hline & $\mathrm{K}$ & 0.85 & 1.07 & 1.12 \\
\hline & $\mathrm{Al}+\mathrm{H}$ & 5.19 & 6.2 & 2.70 \\
\hline & $\mathrm{Al}$ & 4.95 & 5.56 & 2.38 \\
\hline \multirow[t]{4}{*}{8} & Relative proportion of cations & & & \\
\hline & $\mathrm{Ca} / \mathrm{Mg}$ & 0.68 & 0.56 & 2.0 \\
\hline & $\mathrm{Mg} / \mathrm{K}$ & 2.66 & 2.90 & 2.23 \\
\hline & $\mathrm{Ca}+\mathrm{Mg} / \mathrm{K}$ & 4.47 & 4.53 & 6.7 \\
\hline 9 & Acid saturation & 52.7 & 51.2 & 23.9 \\
\hline 10 & ECEC & 9.84 & 12.12 & 11.32 \\
\hline 11 & CEC & 22.18 & 24.13 & 25.08 \\
\hline
\end{tabular}


Hirpa Legesse et al.,

\section{Soil Sampling, pH Calibration and Soil Analysis}

Soil samples were collected from the experimental site at the depth of $0-20 \mathrm{~cm}$ using an auger. Ten soil samples were taken from each arm of the shaped pattern. All samples were bulked and composited and a $1 \mathrm{~kg}$ composite sample was taken for analyzing physical and chemical properties of the soil. The samples were air-dried, disaggregated and sieved through a $2 \mathrm{~mm}$ sieve and analyzed (Thompson and Banerjee, 1991).

The field experiment was conducted with both lime treated and untreated soils. Before liming, the amount of lime required to raise the soil $\mathrm{pH}$ to a level suitable for the growth of common bean was determined. Thus, to calculate the lime requirement of the soil, triplicate dry soil samples each weighing one $\mathrm{kg}$ were thoroughly mixed with $0,400,800$, $1200,1600,2000,2400,2800,3200,3600,4000$ and $4400 \mathrm{mg}$ of calcium carbonate $\left(\mathrm{CaCO}_{3}\right)$. Each soil sample weighing one kilogram was filled in a polyethylene bags having the capacity of three $\mathrm{kg}$ soil. The samples were then thoroughly mixed with the rate of lime to be tested. Then, the samples were saturated with water. When the soil mixed with the lime was well saturated, the mouth of each polyethylene bags filled with the soil-lime mix was closed to avoid evaporation. By the third day, the excess water was drained after opening the mouth of the polyethylene; the samples were incubated under room temperature for a period of four weeks.

Soil $\mathrm{pH}$ was determined in 1:2.5 (w/v) soils to water $\left(\mathrm{H}_{2} \mathrm{O}\right)$ suspension ratio using a glass electrode attracted to a digital $\mathrm{pH}$ meter. Total $\mathrm{N}$ was determined by the micro-kjeldahl procedure as described by Jackson (1958). Available P in the soil samples was determined following the procedure of Bray-II method (Bray and Kurtz, 1945). Organic carbon was determined following the wet digestion method as described by Walkley and Black (1934). Percent organic matter was estimated as: $\% \mathrm{OM}=\%$ organic Carbon X 1.72

Exchangeable potassium was extracted by $1 \mathrm{~N}$ ammonium acetate $\left(1.0 \mathrm{~N} \mathrm{NH}_{4} \mathrm{AOC}\right)$ and the concentration was estimated using a flame photometer after extraction. Cation Exchange Capacity and exchangeable bases were determined by extracting with $1.0 \mathrm{M}$ ammonium acetate at $\mathrm{pH} 7$. Aluminium was determined by titrating with $\mathrm{NaOH}$, and $\mathrm{Ca}$ and $\mathrm{Mg}$ by titration with EDTA. Exchangeable acidity was determined by extracting the soil samples with $1 \mathrm{M} \mathrm{KCl}$ solutions and titrating with standardized $\mathrm{NaOH}$ as described by McLean (1965). Effective cation exchange capacity (ECEC) was estimated by summation of exchangeable bases and exchangeable acidity ( $\mathrm{Al}$ and $\mathrm{H})$. Percent acid saturation was calculated as: $\%$ acid saturation $=($ Exchangeable Acidity $/ E C E C){ }^{*} 100$.
Sci. Technol. Arts Res. J., July-Sep 2013, 2(3): 03-15

\section{Planting Material}

Twenty-five common bean varieties and bredlines were evaluated against soil acidity under field condition. The improved genotypes included in the evaluation were genotypes released by Melkassa and Bako Agricultural Research Centres (Table 2). To have a sizable variability among the genotypes, accessions or varieties that are assumed sensitive, resistant, and mildly resistant to soil acidity were included. All the genotypes included in the evaluation have a bushy and semi prostrate growth habit, and vary in seed colour and size. The genotypes were evaluated based on growth, yield, and yield components.

\section{Liming Material}

A lime is an agricultural material capable of neutralizing soil acidity, i.e., increasing soil $\mathrm{pH}$. The lime $\left(\mathrm{CaCO}_{3}\right)$ was thoroughly and evenly distributed to the plots according to the pre-determined rates of the treatment and was worked into the soil four weeks before sowing the seed of the genotypes. The liming material used in this study had a purity of $93.7 \% \mathrm{CaCO}_{3}$. Lime was applied prior to planting at the rate of $2.16 \mathrm{~kg}$ per plot according to the result obtained from the $\mathrm{pH}$ calibration curve (Figure 1). The amount of lime applied was equivalent to 9 tonnes hectare ${ }^{-1}$.

\section{Treatments and Experimental Design}

The treatments consisted of 25 genotypes and two types of soil amendment (lime treated and untreated). The experiment was laid out as a randomized complete block design and replicated two times per treatment. Each plot consisted of three rows of bean plants occupying a $2 \mathrm{~m}$ long distance and a $1.2 \mathrm{~m}$ width. The distance between successive plots and adjacent blocks were $1 \mathrm{~m}$ and $1.5 \mathrm{~m}$, respectively. The spacing between plants and rows was $10 \mathrm{~cm}$ and $40 \mathrm{~cm}$, respectively. The data were reported as the ratio of grain yield in the lime untreated plot to that in the lime treated plot to adjust for differences in yield potential without acid soil stress according to Johnson et al. (1997).

\section{Planting}

Two seeds of common bean genotype were sown at each planting hole. Two weeks after planting, the seedlings were thinned to one per hill, thereby retaining the recommended population of 250,000 common bean plants per hectare. Fertilizer (DAP) was applied at the rate of $92 \mathrm{~kg} \mathrm{ha}^{-1}(49 \mathrm{mg})$ per plot and mixed well with soil at sowing. All other recommended agronomic management practices were applied uniformly to all genotypes grown in the field. The first weeding was done two weeks after germination, and then weeding was performed as required until the plants started flowering. 
Table 2: Common bean genotypes screened for soil acidity tolerance, on field at Nejo, during the 2009/10 cropping season.

\begin{tabular}{|c|c|c|c|c|c|c|c|c|}
\hline No. & Genotype & $\begin{array}{l}\text { Seed } \\
\text { Source }\end{array}$ & $\begin{array}{l}\text { Proven } \\
\text { ance }\end{array}$ & $\begin{array}{l}\text { Growth } \\
\text { habit }\end{array}$ & Adaptation & $\begin{array}{l}\text { Seed } \\
\text { colour }\end{array}$ & $\begin{array}{l}\text { HSW } \\
\text { (g) }\end{array}$ & $\begin{array}{l}\text { Type of } \\
\text { germplasm }\end{array}$ \\
\hline 1 & New Bilfa 38 & 1 & CIAT & III & LSF & pinto & 29.4 & CRL \\
\hline 2 & New Bilfa 57 & 1 & CIAT & ॥ & LSF & cream & 18.8 & CRL \\
\hline 3 & New Bilfa 50 & 1 & CIAT & III & LSF & red & 14.3 & CRL \\
\hline 4 & New Bilfa 60 & 1 & CIAT & III & LSF & & 21.4 & CRL \\
\hline 5 & New Bilfa 58 & 1 & CIAT & III & LSF & yellow & 27.9 & CRL \\
\hline 6 & Gabisa (Vax-2) & 2 & CIAT & II & wo & $\tan$ & 22.5 & $\mathrm{R}$ \\
\hline 7 & New Bilfa 51 & 1 & CIAT & III & LSF & black & 17.2 & $\mathrm{CRL}$ \\
\hline 8 & New Bilfa 61 & 1 & CIAT & III & LSF & dark & 24.0 & CRL \\
\hline 9 & New Bilfa 45 & 1 & CIAT & II & LSF & black & 19.4 & CRL \\
\hline 10 & New Bilfa 46 & 1 & CIAT & II & LSF & red & 19.5 & $\mathrm{CRL}$ \\
\hline 11 & Awash 1 & 1 & CIAT & II & CRV & white & 14.2 & $\mathrm{R}$ \\
\hline 12 & Awash Melka(PAN182) & 1 & CIAT & II & AAE & White & 12.4 & $\mathrm{R}$ \\
\hline 13 & Argane & 1 & CIAT & II & CRV \& SA & White & 13.7 & $\mathrm{R}$ \\
\hline 14 & Nasser (Dicta-105) & 1 & CIAT & III & AAE & Red & 18.0 & $\mathrm{R}$ \\
\hline 15 & Dimitu (DOR-554) & 1 & CIAT & II & AAE & Red & 16.4 & $\mathrm{R}$ \\
\hline 16 & Dinknesh & 1 & CIAT & II & CRV \& SA & Red & 17.8 & $\mathrm{R}$ \\
\hline 17 & Roba 1 & 1 & CIAT & II & AAE & Cream & 18.9 & $\mathrm{R}$ \\
\hline 18 & Beshbesh(melk97) cross 5 & 1 & CIAT & II & SE & Cream & 13.9 & $\mathrm{R}$ \\
\hline 19 & Anger(EMP-376) & 2 & CIAT & I & BA & Dark red & 18.4 & $\mathrm{R}$ \\
\hline 20 & Chore (STTT-165-92) & 1 & CIAT & II & $R V, S, S W \& E$ & White & 15.2 & $\mathrm{R}$ \\
\hline 21 & SER 16 & 1 & CIAT & II & DT. & Red & 17.9 & bred \\
\hline 22 & SEA 5 & 1 & CIAT & II & DT & cream & 22 & bred \\
\hline 23 & SER78 & 1 & CIAT & II & DT & Dark red & 18.5 & bred \\
\hline 24 & SER 128 & 1 & CAIT & II & DT. & Dark red & 21.0 & bred \\
\hline 25 & SER 176 & 1 & CIAT & II & DT & Red & 17.0 & bred \\
\hline
\end{tabular}

I-Determinate bushy; II = indeterminate bushy; III- indeterminate prostrate; IV = indeterminate climbing, 1 = Melkassa Agricultural Research Centre; 2= Bako Agricultural Research Center CIAT = International Centre for Tropical Agriculture,. HSW- hundred seed weight, CRV-Central Rift Valley, DT-drought tolerant, SE=south Ethiopia, BA- Bako Area, AAE- across all Environments ,R- cross line, RV- rift valley, S- south, SW- south western, WO- western oromiya, LSF- low soil fertility, Rreleased.

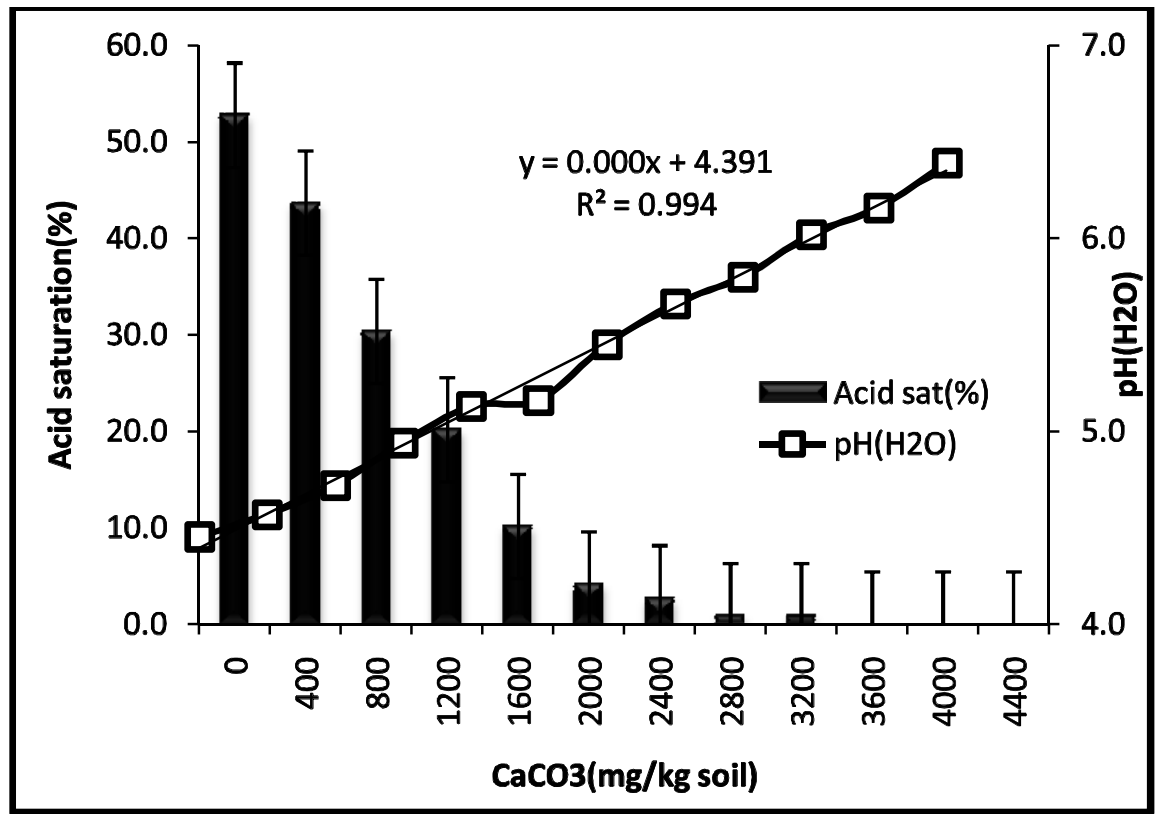

Figure 1: $\mathrm{pH}(\mathrm{a})$ and acid saturation (b) determined through incubation at different rates of lime applied to the soil. 
Hirpa Legesse et al.,

\section{Data Collection and Measurements}

Date of $50 \%$ flowering (number of days from planting to when $50 \%$ of plants in field had at least one open flower), and days to physiological maturity (number of days from planting to when $75 \%$ plants in a field had at least $90 \%$ of their pods dried (Tesso, 2007) were recorded. Plant height and leaf area were recorded from central rows of five plants taken from each plot just at flowering. LAI was calculated as the ratio of total leaf area to ground area occupied by the plant. Leaf area was determined by using CID-202 leaf area meter (CID, Inc., USA).

At harvest, number of pods per plant and the number of primary branches per plant were recorded from five randomly selected plants. The number of seeds per pod and pod length were determined from 10 randomly selected pods from each plot. Grain yield was recorded from the entire plot after counting the number of plants harvested from each plot. Hundred seed weight was determined from 100 seeds randomly sampled from all plants harvested per plot. Harvest index $(\mathrm{HI})$ was calculated as the proportion of seed weight to the above ground dry weight (stem + leaves + pod + seed) at harvest. Pod harvest index was calculated as the weight of dry pod (seed + pod shell) divided by total above ground biomass (Setegn, 2006). Eventually, based on the results of the data analysis, the genotypes were compared and grouped in to sensitive and tolerant ones.

\section{Statistical Analysis}

Data were subjected to analysis of variance using the GLM procedure of SAS software (SAS, 2004). Genotypes were considered as fixed effects whereas replications were reckoned as random effects in the statistical model. The PROC CORR from SAS was used to calculate the correlation of genotypic means. Treatment means that exhibited significant differences were separated using the student-Neuman Keuls test (SNK) at 5\% level of significance (SAS, 2004).

\section{RESULTS}

\section{Pre-cropping Soil Fertility Status and pH Curve}

The physical and chemical properties of the experimental soil are shown in Table 1. Analysing the particle size distribution of the soil revealed the textural class of loamy sand. The soil is very strongly acidic, has high contents of organic matter, total nitrogen and low content of available phosphorus before planting the seeds. Exchangeable $\mathrm{Ca}$ and $\mathrm{Mg}$ in the soil were low and medium, respectively, where as exchangeable $\mathrm{K}$ was high in this soil. The aluminium content of the soil was very high, while the ratio of cations, $\mathrm{Ca} / \mathrm{Mg}$,
Sci. Technol. Arts Res. J., July-Sep 2013, 2(3): 03-15

$\mathrm{Mg} / \mathrm{K}$, and $\mathrm{Ca}+\mathrm{Mg} / \mathrm{K}$ was low for the three cations. In addition, the experimental soil had a high exchangeable acidity and acid saturation (Table 1). The result obtained from the $\mathrm{pH}$ curve indicated that soil $\mathrm{pH}\left(\mathrm{H}_{2} \mathrm{O}\right)$ increased in response to the applied lime, and resulted in decreased acid saturation (Figure 1). From the result obtained, application of lime at the rate of $2000 \mathrm{mg} \mathrm{CaCO}_{3} / \mathrm{kg}\left(9 \mathrm{t} \mathrm{ha}^{-1}\right.$ lime $)$ soil led to increase in soil $\mathrm{pH}$ to optimum level for common bean growth (Fageria et al., 1997). Therefore, this rate was selected to treat the whole experimental soil. At this rate of lime, the $\mathrm{pH}$ of the soil increased from 4.45 to 5.4 , the exchangeable acidity reduced to 0.39 from 5.19 and acid saturation dropped from 52.7 to below $4.13 \%$, which are optimum for common bean growth (Figure 1).

\section{Effect of Soil Acidity on Phenology and Growth}

Phenological stages and growth parameters of common bean genotypes grown under lime treated and untreated soils are indicated in Table 3 . Soil acidity had a marked influence on common bean maturity and growth. Common bean genotypes showed significant differences in days to pod setting, maturity, plant height and leaf area index in response to being grown under both lime treated and untreated soils. The variation was highly significant $(P<0.01)$ for all parameters except for days to flowering in lime untreated soil. Plants of all genotypes were significantly taller and had higher values of leaf area index, but lower number of days to flowering and maturity when grown in lime treated than in untreated soil (Table 3). These results signified that application of lime hastened flowering and maturity of the plants whilst increasing plant height and leaf area (Table 4). On average, the genotypes reached days to $50 \%$ flowering significantly earlier when lime treated than when they were lime untreated. Thus, plants grown in lime treated soil reached days to $50 \%$ flowering earlier than plants grown in the lime untreated soil by about $9 \%$. Similarly, plants grown in the lime treated soil were quicker to reach physiological maturity by about $15.5 \%$ than plants grown in the lime untreated soil.

Gabisa, New Bilfa 61, and SER 16 reached physiological maturity significantly earlier under lime treated than under untreated soils. However, genotypes Dimtu and Beshbesh, reached physiological maturity significantly earlier under lime untreated soil condition than under lime treated condition. The maturity times of all other genotypes were in statistical parity under lime treated and untreated soils (Table 4). The results of this study revealed that the bred lines matured early whereas the improved ones matured relatively late. 
Table 3: Mean squares of phenological stages, growth, yield and yield components of common bean genotypes grown on limed and unlimed soil on field.

\begin{tabular}{|c|c|c|c|c|}
\hline Source of variation & Liming & Genotypes & ERROR & Total \\
\hline \multicolumn{5}{|l|}{ Parameters } \\
\hline \multirow[t]{2}{*}{ Days to $\mathbf{5 0 \%}$ flowering } & UL & $44.33^{\mathrm{NS}}$ & 24.24 & 1646 \\
\hline & $\mathrm{L}$ & $6.83^{* \pi *}$ & 0.77 & 182.5 \\
\hline \multirow[t]{2}{*}{ Days to $\mathbf{5 0} \%$ pod setting } & UL & $87.25^{*}$ & 38.21 & 3011.4 \\
\hline & $\mathrm{L}$ & $21.39^{* * * *}$ & 5.25 & 642.02 \\
\hline \multirow[t]{2}{*}{ Days to $50 \%$ maturity } & UL & $43.4^{* \pi *}$ & 7.06 & 1212.02 \\
\hline & L & $32.47^{\mathrm{xN}}$ & 4.37 & 884.3 \\
\hline \multirow[t]{2}{*}{ LAI } & UL & $0.998^{* * *}$ & 0.281 & 30.68 \\
\hline & $\mathrm{L}$ & $1.03^{*}$ & 0.46 & 37.47 \\
\hline \multirow[t]{2}{*}{ Plant height(cm) } & UL & $312.7^{* x *}$ & 78.1 & 10058.7 \\
\hline & L & $416.3^{*}$ & 100.1 & 12492.5 \\
\hline \multirow[t]{2}{*}{ Branch number per plant } & UL & $0.33^{\text {ns }}$ & 0.25 & 14.28 \\
\hline & L & $0.857^{*}$ & 0.358 & 37.55 \\
\hline \multirow[t]{2}{*}{ Number of pods per plant } & UL & $27.23^{* * *}$ & 2.17 & 706.95 \\
\hline & L & $37.3^{* * * x}$ & 35.8 & 5.01 \\
\hline \multirow[t]{2}{*}{ Number of seeds per pod } & UL & $0.77^{\mathrm{NS}}$ & 0.51 & 30.9 \\
\hline & $\mathrm{L}$ & $1.02^{x * x}$ & 0.277 & 31 \\
\hline \multirow[t]{2}{*}{ Pod length } & UL & $2.18^{* * * x}$ & 0.33 & 60.9 \\
\hline & L & $1.05^{* x *}$ & 0.37 & 34.4 \\
\hline \multirow[t]{2}{*}{100 seed weight } & UL & $34.85^{\mathrm{xm}}$ & 0.96 & 861.2 \\
\hline & $\mathrm{L}$ & $34.89^{* * *}$ & 1.13 & 866.73 \\
\hline \multirow[t]{2}{*}{ Grain Yield (g/P) } & UL & 10.67 & 0.557 & 270.9 \\
\hline & $\mathrm{L}$ & $13.5^{* * *}$ & 0.895 & 347.02 \\
\hline \multirow[t]{2}{*}{ Grain Yield (t/ha) } & UL & $0.667^{* * *}$ & 0.035 & 16.89 \\
\hline & $\mathrm{L}$ & $0.849^{\mathrm{nax}}$ & 0.056 & 27.72 \\
\hline \multirow[t]{2}{*}{ Biomass Yield (t/ha) } & UL & $1.18^{\text {ns }}$ & 0.45 & 40.3 \\
\hline & $\mathrm{L}$ & $1.61^{\mathrm{ns}}$ & 0.983 & 68.75 \\
\hline \multirow[t]{2}{*}{ Pod harvest index } & UL & $0.154^{* *}$ & 0.037 & 4.69 \\
\hline & $\mathrm{L}$ & $0.087^{\text {ns }}$ & 0.056 & 3.88 \\
\hline \multirow[t]{2}{*}{ Harvest index } & UL & $0.0149^{* *}$ & 0.0011 & 0.386 \\
\hline & L & $0.0073^{* * *}$ & 0.002 & 0.22 \\
\hline Relative yield & & $219.9^{*}$ & 86.4 & 7474.2 \\
\hline
\end{tabular}

Table 4: Phenology and growth of common bean genotypes grown under limed and unlimed soil at Nedjo on field during 2009/10 main cropping season.

\begin{tabular}{|c|c|c|c|c|c|c|c|c|}
\hline \multirow{2}{*}{ Genotypes } & \multicolumn{2}{|c|}{ DF } & \multicolumn{2}{|c|}{ DM } & \multicolumn{2}{|c|}{ LAI } & \multicolumn{2}{|c|}{$\mathrm{PH}(\mathrm{cm})$} \\
\hline & UL & $\mathbf{L}$ & UL & $\mathbf{L}$ & UL & $\mathbf{L}$ & UL & $\mathbf{L}$ \\
\hline NB 38 & $54.0^{\dagger}$ & $44.0^{\mathrm{ns}}$ & $91.0^{C}$ & $75.5^{\text {bcd }}$ & $5.72^{a}$ & $4.40^{\mathrm{ab}}$ & $44.6^{\mathrm{bc}}$ & $52.3^{\mathrm{abcd}}$ \\
\hline NB57 & $58.0^{\text {bcd }}$ & $55.0^{\mathrm{ns}}$ & $87.5^{c}$ & $79.5^{\mathrm{bcd}}$ & $3.80^{\mathrm{ab}}$ & $3.60^{\mathrm{ab}}$ & $48.2^{\mathrm{bc}}$ & $66.4^{\mathrm{abcd}}$ \\
\hline NB 50 & $62.0^{\mathrm{a}}$ & $57.0^{\mathrm{ns}}$ & $91.0^{C}$ & $83.5^{\mathrm{bc}}$ & $3.73^{\mathrm{ab}}$ & $5.60^{\mathrm{ab}}$ & $30.4^{c}$ & $41.5^{\mathrm{cd}}$ \\
\hline NB 60 & $55.5^{\text {cdef }}$ & $51.0^{\mathrm{ns}}$ & $92.0^{\mathrm{C}}$ & $77.5^{\mathrm{bcd}}$ & $2.74^{\mathrm{b}}$ & $4.30^{\mathrm{ab}}$ & $56.3^{\mathrm{abc}}$ & $78.3^{\mathrm{abc}}$ \\
\hline NB58 & $54.5^{\mathrm{ef}}$ & $56.5^{\mathrm{ns}}$ & $90.5^{c}$ & $81.0^{\mathrm{bcd}}$ & $3.94^{\mathrm{ab}}$ & $3.80^{\mathrm{ab}}$ & $57.7^{\mathrm{abc}}$ & $84.5^{\mathrm{a}}$ \\
\hline Gabisa & $58.0^{\text {bcd }}$ & $53.5^{\mathrm{ns}}$ & $103.0^{a}$ & $80.5^{\mathrm{bcd}}$ & $3.31^{\mathrm{ab}}$ & $3.95^{\mathrm{ab}}$ & $39.1^{\mathrm{bc}}$ & $59.3^{\mathrm{abcd}}$ \\
\hline NB51 & $58.0^{\text {bcd }}$ & $54.5^{\mathrm{ns}}$ & $90.5^{c}$ & $82.0^{\text {bcd }}$ & $4.93^{\mathrm{ab}}$ & $5.50^{\mathrm{ab}}$ & $53.9^{\mathrm{abc}}$ & $57.9^{\mathrm{abcd}}$ \\
\hline NB 61 & $57.0^{\text {bcdet }}$ & $49.0^{\text {ns }}$ & $94.0^{\mathrm{bc}}$ & $72.0^{d}$ & $3.10^{\mathrm{ab}}$ & $3.35^{\mathrm{b}}$ & $60.1^{\mathrm{abc}}$ & $63.1^{\mathrm{abcd}}$ \\
\hline NB 45 & $56.0^{\text {cdef }}$ & $52.0^{\mathrm{ns}}$ & $88.5^{c}$ & $76.0^{\text {bcd }}$ & $3.71^{\mathrm{ab}}$ & $5.55^{\mathrm{ab}}$ & $37.0^{\mathrm{bc}}$ & $42.7^{\mathrm{bcd}}$ \\
\hline NB 46 & $56.0^{\text {cdef }}$ & $55.0^{\mathrm{ns}}$ & $92.5^{\mathrm{c}}$ & $81.5^{\mathrm{bcd}}$ & $3.67^{\mathrm{ab}}$ & $4.25^{\mathrm{ab}}$ & $38.9^{\mathrm{bc}}$ & $43.2^{\mathrm{bcd}}$ \\
\hline Awash 1 & $56.5^{\text {bcdet }}$ & $47.5^{\mathrm{ns}}$ & $90.0^{c}$ & $77.0^{\mathrm{bcd}}$ & $5.01^{\mathrm{ab}}$ & $3.80^{\mathrm{ab}}$ & $67.7^{\mathrm{ab}}$ & $70.2^{\mathrm{abcd}}$ \\
\hline Awash M. & $56.5^{\text {bcdef }}$ & $41.5^{\mathrm{ns}}$ & $90.5^{c}$ & $81.5^{\mathrm{bcd}}$ & $4.82^{\mathrm{ab}}$ & $4.90^{\mathrm{ab}}$ & $37.3^{\mathrm{bc}}$ & $39.7^{\mathrm{cd}}$ \\
\hline Argane & $55.0^{\text {def }}$ & $53.5^{\mathrm{ns}}$ & $89.5^{c}$ & $78.0^{\text {bcd }}$ & $3.87^{\mathrm{ab}}$ & $4.30^{\mathrm{ab}}$ & $35.1^{\mathrm{bc}}$ & $46.9^{\mathrm{abcd}}$ \\
\hline Nasser & $55.5^{\text {cdet }}$ & $50.5^{\text {ns }}$ & $89.0^{\mathrm{C}}$ & $75.5^{\mathrm{bcd}}$ & $4.29^{a b}$ & $4.20^{\mathrm{ab}}$ & $79.9^{\mathrm{a}}$ & $81.7^{\mathrm{ab}}$ \\
\hline Dimitu & $56.5^{\text {bcdef }}$ & $58.0^{\mathrm{ns}}$ & $90.0^{C}$ & $91.5^{\mathrm{a}}$ & $3.52^{\mathrm{ab}}$ & $4.25^{\mathrm{ab}}$ & $46.2^{\mathrm{bc}}$ & $67.4^{\mathrm{abcd}}$ \\
\hline Dinknesh & $59.5^{\mathrm{b}}$ & $56.5^{\mathrm{ns}}$ & $90.5^{\mathrm{c}}$ & $83.0^{\mathrm{bc}}$ & $3.99^{a b}$ & $4.50^{\mathrm{ab}}$ & $44.2^{\mathrm{bc}}$ & $51.9^{\mathrm{abcd}}$ \\
\hline Roba 1 & $58.5^{\mathrm{bc}}$ & $56.0^{\text {ns }}$ & $94.5^{\mathrm{bc}}$ & $82.0^{\text {bcd }}$ & $4.16^{\mathrm{ab}}$ & $4.05^{\mathrm{ab}}$ & $35.7^{\mathrm{bc}}$ & $37.5^{\mathrm{d}}$ \\
\hline Beshbesh & $57.5^{\text {bcde }}$ & $56.5^{\mathrm{ns}}$ & $90.5^{\mathrm{c}}$ & $86.0^{\mathrm{ab}}$ & $3.35^{\mathrm{ab}}$ & $4.55^{\mathrm{ab}}$ & $42.2^{\mathrm{bc}}$ & $52.2^{\mathrm{abcd}}$ \\
\hline Anger & $57^{\text {bcdet }}$ & $54.5^{\mathrm{ns}}$ & $99.0^{\mathrm{ab}}$ & $77.5^{\mathrm{bcd}}$ & $4.61^{\mathrm{ab}}$ & $4.35^{\mathrm{ab}}$ & $39.4^{\mathrm{bc}}$ & $47.1^{\mathrm{abcd}}$ \\
\hline Chore & $57.0^{\text {bcdef }}$ & $58.5^{\mathrm{ns}}$ & $100.5^{a}$ & $85.0^{a b c}$ & $4.64^{\mathrm{ab}}$ & $4.54^{\mathrm{ab}}$ & $30.6^{c}$ & $35.9^{d}$ \\
\hline SER 16 & $54.5^{\mathrm{et}}$ & $44.0^{\mathrm{ns}}$ & $87.5^{c}$ & $71.5^{\mathrm{d}}$ & $5.14^{\mathrm{ab}}$ & $4.65^{\mathrm{ab}}$ & $50.5^{\mathrm{bc}}$ & $53.8^{\mathrm{abcd}}$ \\
\hline SEA 5 & $55.0^{\text {def }}$ & $52.0^{\mathrm{ns}}$ & $87.0^{\mathrm{C}}$ & $76.5^{\text {bcd }}$ & $3.03^{\mathrm{ab}}$ & $6.20^{\mathrm{a}}$ & $33.9^{\mathrm{bc}}$ & $47.9^{\mathrm{abcd}}$ \\
\hline SER 78 & $55.5^{\text {cdef }}$ & $49.0^{\mathrm{ns}}$ & $90.5^{c}$ & $74.5^{\mathrm{dc}}$ & $4.98^{a b}$ & $4.70^{\mathrm{ab}}$ & $38.9^{\mathrm{bc}}$ & $41.3^{\mathrm{cd}}$ \\
\hline SER 128 & $54.5^{\text {et }}$ & $46.5^{\mathrm{ns}}$ & $88.5^{c}$ & $74.5^{\mathrm{dc}}$ & $4.61^{a b}$ & $5.20^{\mathrm{ab}}$ & $64.9^{\mathrm{abc}}$ & $74.5^{\mathrm{abcd}}$ \\
\hline SER 176 & $54.5^{\text {ef }}$ & $49.0^{\mathrm{ns}}$ & $88.0^{\mathrm{C}}$ & $75.5^{\mathrm{bcd}}$ & $5.1^{\mathrm{ab}}$ & $3.83^{\mathrm{ab}}$ & $41.1^{\mathrm{bc}}$ & $47.2^{\mathrm{abcd}}$ \\
\hline Mean & 56.5 & 52 & 91.4 & 79.14 & 3.89 & 4.45 & 46.2 & 55.4 \\
\hline CV (\%) & 1.55 & 9.5 & 2.3 & 3.4 & 13.6 & 15.3 & 19.2 & 18.1 \\
\hline SE+ & 0.88 & 4.9 & 2.09 & 2.66 & 0.53 & 0.68 & 8.84 & 10.01 \\
\hline
\end{tabular}


Hirpa Legesse et al.,

\section{Effect of Soil Acidity on Yield and Yield Components}

Soil acidity had significant effect on yield and yield components of common bean genotypes (Table 3). Highly significant $(P<0.001)$ differences were observed among genotypes for number pods per plant, pod length, grain yield, hundred seed weight and harvest index in both soils regimes. However, non-significant differences were found among common bean genotypes for number of seeds per pod, number branches per plant and biomass yield in lime untreated soil. On average, the genotypes gave higher yield and yield components in lime treated soil (Table 5). SER materials gave higher number of pods per plant but shorter pods in both lime treated and untreated soil (Table 5). Generally, longer pods were harvested from lime treated soil than from untreated soil (Table 5). Thirteen genotypes, namely, Awash 1, Awash Melka, Argane, Nasser, Dimitu, Dinknesh, Anger, Chore, SER 16, SEA 5, SER 78, SER 128, and SER176 gave statistically non-significant pod
Sci. Technol. Arts Res. J., July-Sep 2013, 2(3): 03-15

length. The pod lengths of all other genotypes obtained under both lime treated and untreated soil conditions were in statistical parity.

Under the lime untreated soil condition, the minimum pod length was obtained for the genotype named Chore whereas the maximum was obtained for the genotypes named new BILFA 58. The highest hundred seed weight was recorded for BILFA materials (New Bilfa 38 and New Bilfa 58) both under lime treated and untreated soils. On the other hand, the lowest hundred seed weight was recorded for new BILFA 50, Awash melka, Argane, Beshbesh, Chore and Awash 1 under lime untreated soil (Table 5). However, the released varieties produced generally lower hundred seed weights than often reported by research centres under both lime treated and untreated soils. In general; lime application to the soil increased hundred seed weight of common genotypes by about $3.54 \%$.

Table 5: Yield components of common bean genotypes grown under lime untreated and treated acid soil in field at Nedjo.

\begin{tabular}{|c|c|c|c|c|c|c|c|c|}
\hline \multirow{2}{*}{ Genotypes } & \multicolumn{2}{|c|}{ NPP } & \multicolumn{2}{|c|}{ Pod Length(cm) } & \multicolumn{2}{|c|}{100 S. weight(g) } & \multicolumn{2}{|c|}{$\mathrm{HI}$} \\
\hline & Unlimed & Limed & Unlimed & Limed & Unlimed & Limed & Unlimed & Limed \\
\hline NB 38 & $16^{\text {cdetg }}$ & $18^{\mathrm{ef}}$ & $8.9^{a b c}$ & $10.6^{a}$ & $29.4^{\mathrm{a}}$ & $30.9^{a}$ & $0.42^{\mathrm{bc}}$ & $0.41^{a}$ \\
\hline NB 57 & $18^{\text {bcdet }}$ & $23^{\text {bcaet }}$ & $8.8^{\mathrm{abca}}$ & $10.9^{a}$ & $18.8^{\mathrm{etg}}$ & $17.3^{\operatorname{tgn} 1}$ & $0.34^{\text {caetg }}$ & $0.33^{\mathrm{abca}}$ \\
\hline NB 50 & $19^{\text {bcdet }}$ & $23^{\mathrm{bcde}}$ & $8.4^{\mathrm{abcdet}}$ & $9.4^{\mathrm{ab}}$ & $14.3^{\mathrm{lk} k}$ & $15.9^{\mathrm{ghl}}$ & $0.34^{\text {cdetg }}$ & $0.40^{\mathrm{a}}$ \\
\hline NB 60 & $12^{g n}$ & $14^{\mathrm{et}}$ & $9.1^{\mathrm{abc}}$ & $10.3^{\mathrm{ab}}$ & $21.4^{\text {bcde }}$ & $26.0^{\mathrm{b}}$ & $0.24^{\text {etghı }}$ & $0.32^{\mathrm{abca}}$ \\
\hline NB 58 & $20^{\text {bcde }}$ & $21^{\text {cdet }}$ & $10.0^{a}$ & $10.6^{\mathrm{a}}$ & $27.9^{a}$ & $30.4^{\mathrm{a}}$ & $0.37^{\mathrm{cd}}$ & $0.35^{\mathrm{abcd}}$ \\
\hline Gabisa & $14^{\operatorname{tgn}}$ & $19^{\mathrm{et}}$ & $8.4^{\text {abcdet }}$ & $9.9^{\mathrm{ab}}$ & $22.5^{\mathrm{bc}}$ & $21.7^{\mathrm{cd}}$ & $0.21^{\mathrm{nl}}$ & $0.23^{\mathrm{cd}}$ \\
\hline NB 51 & $18^{\text {bcdet }}$ & $21^{\text {det }}$ & $8.7^{\mathrm{abcde}}$ & $9.8^{\mathrm{ab}}$ & $17.2^{g h i}$ & $18.1^{\text {detghı }}$ & $0.40^{\mathrm{bc}}$ & $0.31^{\mathrm{abcd}}$ \\
\hline NB 61 & $20^{\text {bcde }}$ & $22^{\text {caet }}$ & $8.8^{\text {abcde }}$ & $10^{\mathrm{ab}}$ & $24.0^{\mathrm{b}}$ & $22.4^{\mathrm{c}}$ & $0.32^{\text {caetgn }}$ & $0.33^{\mathrm{abca}}$ \\
\hline NB 45 & $20^{\text {bcde }}$ & $24^{\text {bcde }}$ & $9.2^{\mathrm{abc}}$ & $10.3^{\mathrm{ab}}$ & $19.4^{\operatorname{detg}}$ & $18.4^{\text {detghı }}$ & $0.33^{\text {cdetg }}$ & $0.32^{\mathrm{abcd}}$ \\
\hline NB 46 & $19^{\text {bcaet }}$ & $22^{\text {bcaet }}$ & $7.8^{\text {bcaetg }}$ & $9.1^{a b}$ & $19.5^{\text {aetg }}$ & $18.3^{\text {aetgnı }}$ & $0.35^{\text {caetg }}$ & $0.40^{\mathrm{a}}$ \\
\hline Awash 1 & $15^{\text {cdetgh }}$ & $22^{\text {cdet }}$ & $6.6^{\operatorname{detg}}$ & $8.5^{\mathrm{ab}}$ & $14.2^{1 \mathrm{lk}}$ & $15.0^{\prime}$ & $0.32^{\text {cdetg }}$ & $0.33^{\mathrm{abcd}}$ \\
\hline Awash M. & $20^{\text {bcde }}$ & $24^{\text {bcde }}$ & $8.2^{\text {abcdet }}$ & $8.6^{\mathrm{ab}}$ & $12.4^{\mathrm{k}}$ & $15.4^{\mathrm{nI}}$ & $0.34^{\text {caetg }}$ & $0.38^{\mathrm{abc}}$ \\
\hline Argane & $16^{\text {cdetg }}$ & $23^{\mathrm{bcde}}$ & $8.1^{\text {abcdetg }}$ & $8.1^{\mathrm{b}}$ & $13.7^{\mathrm{k}}$ & $14.7^{1}$ & $0.27^{\operatorname{detgh}}$ & $0.32^{\mathrm{abcd}}$ \\
\hline Nasser & $18^{\text {bcdet }}$ & $24^{\text {bcae }}$ & $6.6^{\text {etg }}$ & $9.1^{\mathrm{ab}}$ & $18.0^{\operatorname{tgn}}$ & $20.0^{\text {caet }}$ & $0.28^{\text {aetgn }}$ & $0.25^{\mathrm{bcd}}$ \\
\hline Dimitu & $15^{\text {etgn }}$ & $18^{\mathrm{et}}$ & $7.6^{\text {bcdetg }}$ & $9.6^{\mathrm{ab}}$ & $16.4^{\text {ghij }}$ & $16.8^{\prime}$ & $0.35^{\text {cdet }}$ & $0.30^{\mathrm{abcd}}$ \\
\hline Dinknesh & $11^{\mathrm{n}}$ & $17^{\mathrm{et}}$ & $6.4^{\mathrm{tg}}$ & $8.9^{\mathrm{ab}}$ & $17.8^{\operatorname{tgn}}$ & $18.6^{\text {detgnı }}$ & $0.15^{1}$ & $0.21^{a}$ \\
\hline Roba 1 & $15^{\operatorname{detgh}}$ & $19^{\mathrm{et}}$ & $7.6^{\text {bcdetg }}$ & $9.6^{\mathrm{ab}}$ & $18.9^{\text {etg }}$ & $19.7^{\text {cdetg }}$ & $0.24^{\text {etghı }}$ & $0.35^{\mathrm{abcd}}$ \\
\hline Beshbesh & $20^{\text {bcae }}$ & $21^{\text {cdet }}$ & $8.0^{\text {abcaetg }}$ & $9.5^{\mathrm{ab}}$ & $13.9^{\mathrm{k}}$ & $17.0^{\operatorname{tgn} ı}$ & $0.35^{\text {caetg }}$ & $0.37^{\mathrm{abca}}$ \\
\hline Anger & $13^{g h}$ & $18^{\mathrm{et}}$ & $7.1^{\text {cdetg }}$ & $9.9^{\mathrm{ab}}$ & $18.4^{\mathrm{etgh}}$ & $19.2^{\operatorname{tgh} 1}$ & $0.22^{\mathrm{ghl}}$ & $0.24^{\mathrm{cd}}$ \\
\hline Chore & $12^{\mathrm{gn}}$ & $17^{\mathrm{et}}$ & $6.1^{g}$ & $9.2^{\mathrm{ab}}$ & $15.2^{\text {nנjk }}$ & $16.8^{\operatorname{tgn} 1}$ & $0.24^{\text {tgnı }}$ & $0.23^{\mathrm{cd}}$ \\
\hline SER 16 & $26^{a}$ & $30^{a b}$ & $7.1^{\text {cdetg }}$ & $9.1^{\mathrm{ab}}$ & $17.9^{\operatorname{tgn}}$ & $17.6^{\text {etghı }}$ & $0.40^{\mathrm{bc}}$ & $0.42^{\mathrm{a}}$ \\
\hline SEA 5 & $22^{\mathrm{D}}$ & $32^{a}$ & $9.6^{\mathrm{ab}}$ & $10^{\mathrm{ab}}$ & $22.0^{\mathrm{bca}}$ & $21.2^{\text {cae }}$ & $0.42^{\mathrm{DC}}$ & $0.39^{\mathrm{ab}}$ \\
\hline SER 78 & $18^{\text {bcdet }}$ & $20^{\text {det }}$ & $7.0^{\text {cdetg }}$ & $8.6^{\mathrm{ab}}$ & $18.5^{\mathrm{etgh}}$ & $18.1^{\text {detghı }}$ & $0.36^{\text {cde }}$ & $0.28^{\mathrm{abcd}}$ \\
\hline SER 128 & $21^{\mathrm{DC}}$ & $29^{a b c}$ & $7.3^{\text {calg }}$ & $9.4^{\mathrm{ab}}$ & $21.0^{\text {cdet }}$ & $19.2^{\text {caetgn }}$ & $0.49^{\mathrm{ab}}$ & $0.33^{\mathrm{abca}}$ \\
\hline SER176 & $21^{\mathrm{bcd}}$ & $28^{\mathrm{abcd}}$ & $8.3^{\mathrm{abcd}}$ & $8.9^{\mathrm{ab}}$ & $17.0^{\mathrm{ghl}}$ & $18.4^{\text {detghı }}$ & $0.52^{\mathrm{a}}$ & $0.33^{\mathrm{abcd}}$ \\
\hline Mean & 17.4 & 21.8 & 7.96 & 9.5 & 18.79 & 19.48 & 0.32 & 0.33 \\
\hline CV (\%) & 8.5 & 10.3 & 7.22 & 6.4 & 5.22 & 5.45 & 9.84 & 11.99 \\
\hline PR (\%) & 20.2 & & 16.2 & & 3.54 & & 3.03 & \\
\hline
\end{tabular}

UL- unlimed, L- Limed, CV- coefficient of variation, Ns - non-significant, SE= standard error, PR- percent reduction [ 1$(\mathrm{UL} / \mathrm{L})]^{\star} 100$. 
Hirpa Legesse et al.,

The effect of lime was greatest for pod number per plant with an average increase of $20.2 \%$ for the lime treated soil than for the untreated soil. Seed number per pod was less sensitive to soil acidity, with a mean increase of only $15.4 \%$ in lime treated soil than untreated soils. Similarly, pod length was reduced by $16.2 \%$ on average, suggesting that reduction in grain yield due to soil acidity stress may have stemmed from the effect on pod number per plant.

Yield responses showed that the grain yield was significantly $(P<0.01)$ affected by lime application. In general, liming resulted in a mean improvement in grain yield for all genotypes over no lime application (Table 3). The magnitude of increase in grain yield

\section{Sci. Technol. Arts Res. J., July-Sep 2013, 2(3): 03-15}

and total dry biomass yield due to liming was 25.7 and $27.6 \%$, respectively over the no lime treatment. Higher absolute grain yield in the lime untreated soil was recorded for the genotypes new BILFA 58, SER 176, SEA 5, and Beshbesh (Figure 2). However, Gabisa, Chore, and Anger produced the lowest absolute grain yields on the lime untreated soil. Soil acidity also affects the harvest index of the genotypes. However, lime application increased harvest index and pod harvest index of the genotypes (Table 6). The highest harvest index was recorded for SER materials (SER 176 and SER 128) both under lime untreated and treated soil condition. The lowest harvest index was recorded for Dinknesh, Gabisa, Roba 1, Anger and Chore, in lime untreated soil.

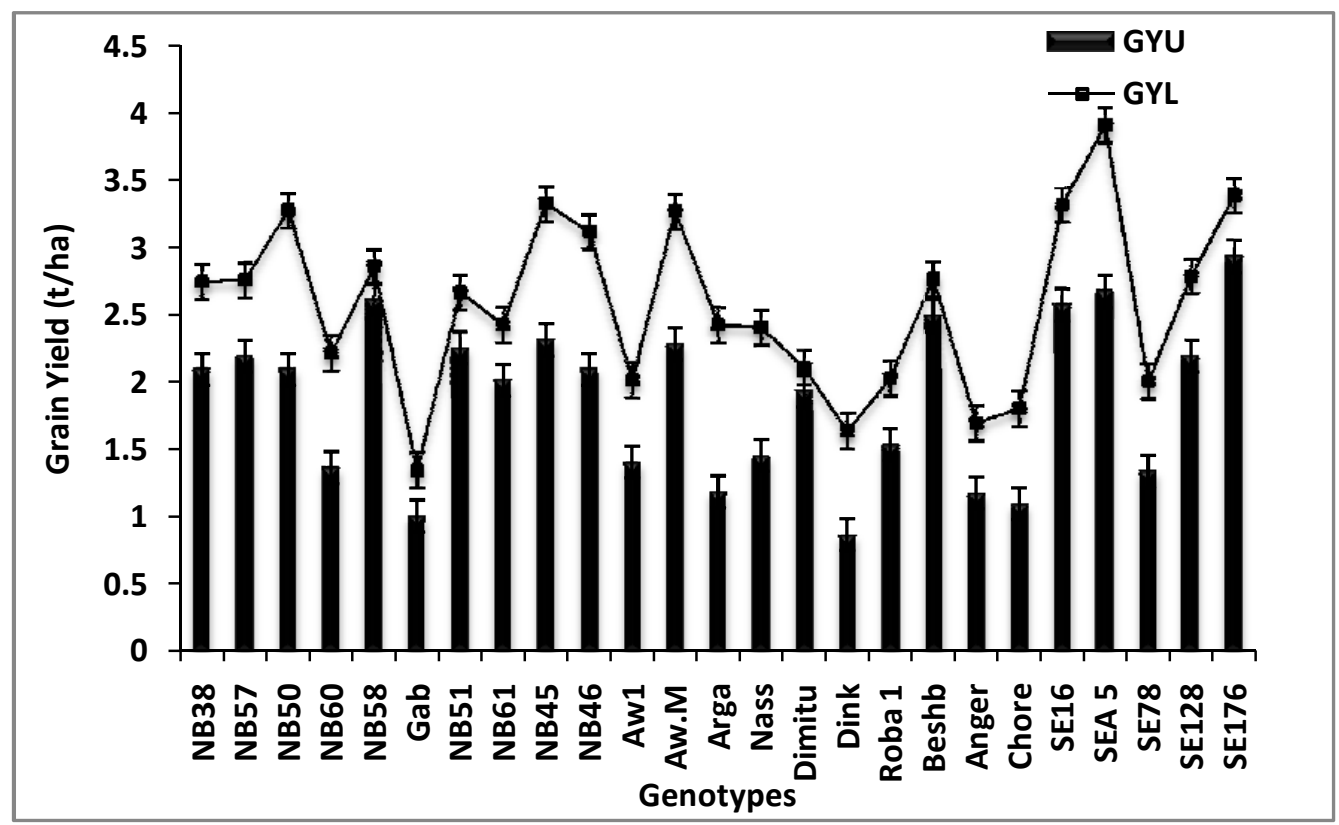

Figure 2: Grain Yield (GY) of common bean genotypes tested for soil acidity tolerance under lime (L) treated and untreated $(U)$ soil at Nedjo, Western Ethiopia.

Table 6: Average values of Grain yield, Biomass, pod harvest index $(\mathrm{PHI})$, Harvest index $(\mathrm{HI})$ \& hundred seed weight (HSW) of common bean genotype grown under lime treated and untreated soils at Nedjo.

\begin{tabular}{llllllllll}
\hline Treatment & DF & DP & DM & LAl & PH & BN & NPP & NSP & PL \\
\hline Unlimed & $56.5^{\mathrm{a}}$ & $70.9^{\mathrm{a}}$ & $91.4^{\mathrm{a}}$ & $3.9^{\mathrm{b}}$ & $46.1^{\mathrm{b}}$ & $5.3^{\mathrm{b}}$ & $17.4^{\mathrm{b}}$ & $4.7^{\mathrm{b}}$ & $7.9^{\mathrm{b}}$ \\
Limed & $52.0^{\mathrm{b}}$ & $64.8^{\mathrm{b}}$ & $79.2^{\mathrm{b}}$ & $4.5^{\mathrm{a}}$ & $55.4^{\mathrm{a}}$ & $6.7^{\mathrm{a}}$ & $21.8^{\mathrm{a}}$ & $5.5^{\mathrm{b}}$ & $9.5^{\mathrm{a}}$ \\
PR & 8.7 & 9.3 & 15.5 & 12.6 & 16.7 & 21.8 & 20.2 & 14.6 & 16.3 \\
Mean & 54.3 & 67.8 & 85.3 & 4.2 & 50.8 & 5.99 & 19.6 & 5.1 & 8.7 \\
CV (\%) & 6.5 & 6.8 & 2.8 & 14.8 & 18.7 & 10.80 & 9.6 & 12.2 & 6.7 \\
\hline Treatment & $\mathrm{GY}(\mathrm{g} / \mathrm{p})$ & $\mathrm{GY}(\mathrm{t} / \mathrm{ha})$ & $\mathrm{BY}(\mathrm{t} / \mathrm{ha})$ & $\mathrm{PHI}$ & $\mathrm{HI}$ & $\mathrm{HSW}$ & & & \\
Unlimed & $7.4^{\mathrm{b}}$ & $1.9^{\mathrm{b}}$ & $3.6^{\mathrm{b}}$ & $1.01^{\mathrm{a}}$ & $0.33^{\mathrm{a}}$ & $18.8^{\mathrm{b}}$ & & & \\
Limed & $10.3^{\mathrm{a}}$ & $2.6^{\mathrm{a}}$ & $5.3^{\mathrm{a}}$ & $1.05^{\mathrm{a}}$ & $0.34^{\mathrm{a}}$ & $19.5^{\mathrm{a}}$ & & & \\
PR & 8.9 & 2.3 & 4.6 & 22.9 & 0.33 & 19.1 & & & \\
Mean & 25.7 & 25.7 & 27.6 & 3.81 & 2.9 & 3.5 & & & \\
CV (\%) & 9.6 & 9.5 & 19.97 & 1.03 & 11.6 & 5.3 & & & \\
\hline
\end{tabular}

Where, $\mathrm{GY}=$ grain yield, $\mathrm{DM}=$ dry matter, $\mathrm{PHI}=$ pod harvest Index, $\mathrm{HI}=$ Harvest index, $\mathrm{HSW}=$ hundred seed weight $(\mathrm{g})$, t/ha= tonne per hectare $\mathrm{CV}=$ coefficient variation 
Hirpa Legesse et al.,

\section{Relative vs. Absolute Yield}

Relative grain yields and percent yield reductions of the genotypes in response to being grown in lime treated and untreated soils are shown in Figure 3. The variations in relative yields of common bean genotypes were significant (Table 3). The genotypes produced significantly higher yields under lime treated soil than under untreated soil, exceeding the yield obtained in the lime untreated soil by about $7.7-47.3 \%$. On average, the genotypes produced $74 \%$ relative grain yield when

\section{Sci. Technol. Arts Res. J., July-Sep 2013, 2(3): 03-15}

grown on lime untreated soil than when grown in lime treated soil. Thus, on average the grain yields of the genotypes reduced by about $26 \%$ in response to growing in lime untreated soil. Higher relative grain yields were recorded for Dimitu, new BILFA 58, Beshbesh, SER 176, new BILFA 51 and new BILFA 61 genotypes. However, Dinknesh, Chore, Nasser and new BILFA 60 produced very low grain yields or the grain yields suffered most reductions in response to growing them in the lime untreated soil (Figure 3).

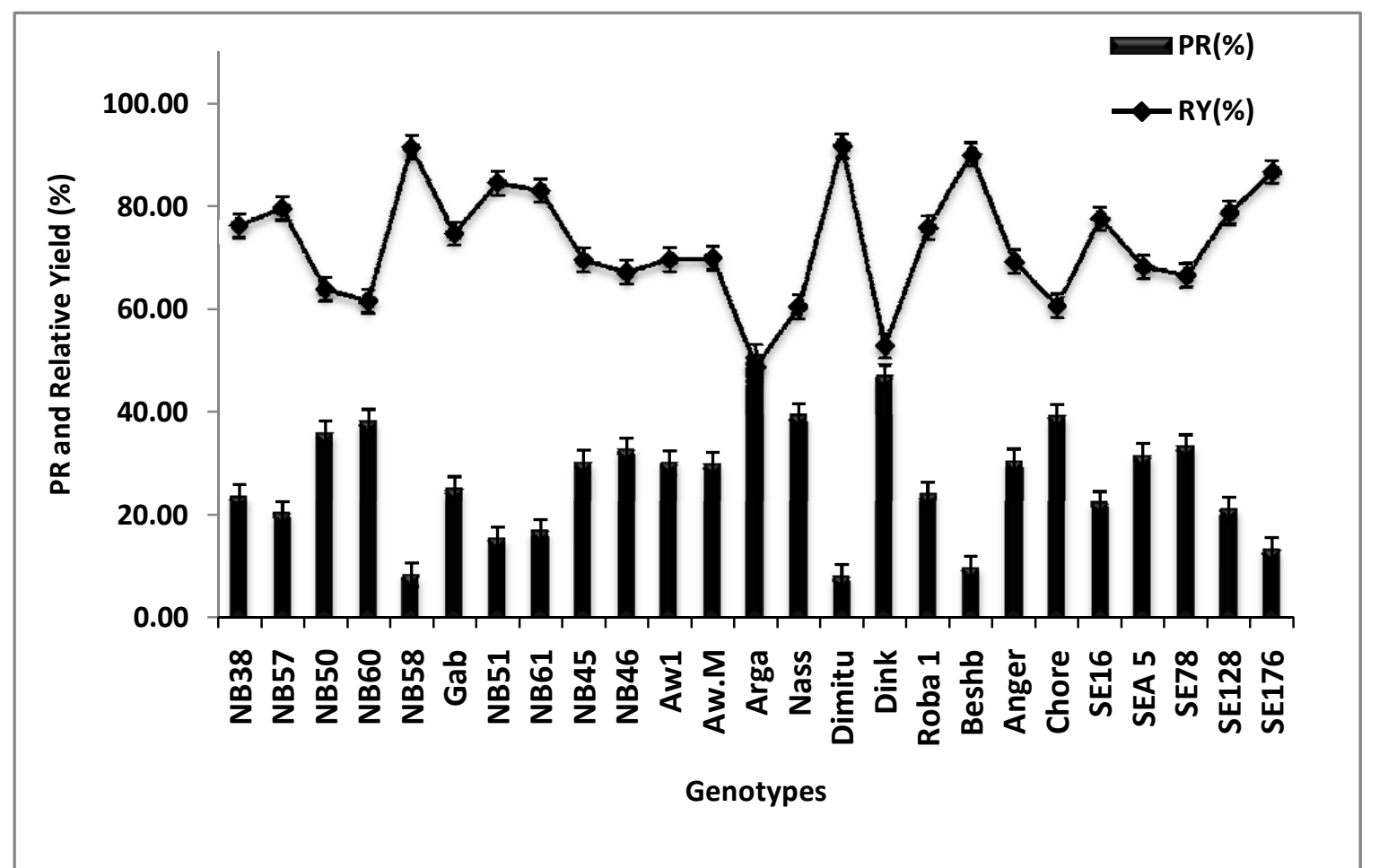

Figure 3: Relative Yield (RY\%), and percent reduction (PR) of common bean genotypes tested for soil acidity tolerance under lime treated and untreated soil at Nedjo, Western Ethiopia.

\section{Correlation Analysis}

The correlation coefficients between some measured traits (selected pairs of parameters) are presented in Table 7 for lime treated and untreated soils. Correlation analysis indicated that there was a positive and significant correlation between days to flowering and days to maturity in both soil regimes. Days to flowering and maturity had also significant and positive correlations with the number of branches per plant (Table 7). However, days to flowering had a strong and negative correlation with number of pods per plant, and harvest index under both lime treated and untreated soil conditions. Days to maturity had a strong and negative relation with number of pods per plant, grain yield, pod harvest index and harvest index in soil not treated with lime (Table 7). Pair wise correlation analysis indicated a strong and positive relation of grain yield with number of pods per plant, number of seeds per pod, and pod length in lime untreated soil (Table 7).
Similarly, grain yield of common bean genotypes were highly and positively correlated with the number of pods per plant and number of seeds per pod in lime treated soil (Table 7). Number of pods per plant was positively correlated with plant height in both lime treated and untreated soils. However, the correlation between plant height and pod length was weak and negative in lime untreated soil. In both lime treated and untreated soils 100 seed weight was strongly and positively correlated with pod length and weakly correlated with the number of pods in lime untreated soil. However, 100 seed weight was weakly and negatively correlated with number of seeds per pod in both liming regimes soil. Harvest index was negatively correlated with physiological maturity in both soils. However, harvest index was strongly and positively correlated with number of pods per plant, number of seeds per pod, and grain yield and negatively correlated with biomass yield in both soil types (Table 7). 
Table 7: Pearson correlation analysis for growth, yield and yield components of common bean genotypes grown under lime untreated (1st) and lime treated (2nd) soil on field at Nedjo.

\begin{tabular}{|c|c|c|c|c|c|c|c|c|c|c|c|c|c|c|}
\hline & DF & DM & LAI & $\mathbf{P H}$ & BN & NPP & NSP & PL & $\mathbf{G Y}$ & RY & BY & PHI & $\mathrm{HI}$ & HSW \\
\hline DF & 1 & 0.32 & -0.21 & -0.30 & $0.42^{*}$ & -0.34 & 0.06 & -0.15 & -0.35 & -0.24 & 0.37 & -0.13 & $-0.49^{*}$ & -0.39 \\
\hline DM & & 1 & 0.09 & -0.28 & 0.11 & $-0.58^{* *}$ & -0.28 & -0.22 & $-0.64^{* *}$ & -0.19 & 0.08 & $-0.50^{*}$ & $-0.60^{* *}$ & 0.09 \\
\hline LAI & & & 1 & 0.04 & 0.01 & 0.06 & -0.02 & $-0.48^{*}$ & -0.02 & -0.09 & $-0.56^{* *}$ & 0.05 & 0.31 & -0.19 \\
\hline PH & & & & 1 & -0.30 & 0.10 & -0.14 & -0.12 & -0.02 & 0.3 & -0.24 & -0.05 & 0.13 & 0.23 \\
\hline BN & & & & & 1 & -0.07 & -0.13 & 0.09 & 0.08 & 0.14 & 0.21 & 0.19 & -0.06 & -0.07 \\
\hline NPP & & & & & & 1 & $0.48^{*}$ & 0.35 & $.0 .84^{* * *}$ & $0.42^{*}$ & -0.14 & $0.66^{\star \star \star}$ & $0.75^{* * *}$ & 0.08 \\
\hline NSP & & & & & & & 1 & 0.39 & $0.54^{* *}$ & 0.37 & -0.15 & $0.54^{* *}$ & 0.51 & -0.08 \\
\hline PL & & & & & & & & 1 & $0.59^{* *}$ & $0.43^{*}$ & 0.20 & $0.54^{* *}$ & 0.34 & $0.51^{* *}$ \\
\hline GY & & & & & & & & & 1 & $0.62^{* \star \star}$ & -0.05 & $0.80^{* \star *}$ & $0.83^{* * *}$ & 0.09 \\
\hline RY & & & & & & & & & & 1 & -0.08 & $0.46^{*}$ & $0.54^{\star *}$ & 0.17 \\
\hline BY & & & & & & & & & & & 1 & -0.26 & $-0.59^{* *}$ & -0.16 \\
\hline PHI & & & & & & & & & & & & 1 & $0.76^{* * *}$ & 0.13 \\
\hline HI & & & & & & & & & & & & & 1 & 0.16 \\
\hline HSW & & & & & & & & & & & & & & 1 \\
\hline & DF & DM & LAI & $\mathbf{P H}$ & BN & NPP & NSP & PL & GY & BY & PHI & HI & HSW & \\
\hline$\overline{D F}$ & 1 & 0.69 & 0.07 & -0.07 & 0.47 & -0.42 & 0.03 & 0.19 & 0.32 & 0.09 & -0.29 & -0.37 & -0.12 & \\
\hline DM & & 1 & -0.08 & -0.13 & $0.42^{*}$ & $-0.47^{\pi}$ & -0.13 & -0.01 & -0.24 & -0.12 & -0.04 & -0.16 & -0.24 & \\
\hline LAI & & & 1 & -0.32 & 0.16 & $0.42^{*}$ & 0.39 & -0.11 & $0.44^{*}$ & $0.40^{*}$ & -0.07 & 0.14 & $-0.40^{*}$ & \\
\hline $\mathrm{PH}$ & & & & 1 & -0.07 & -0.05 & -0.37 & 0.33 & -0.13 & 0.05 & -0.20 & -0.12 & $0.48^{\star}$ & \\
\hline BN & & & & & 1 & -0.11 & 0.08 & -0.16 & -0.16 & 0.39 & -0.33 & -0.39 & -0.30 & \\
\hline NPP & & & & & & 1 & $0.53^{\pi x}$ & -0.17 & $0.77^{\pi \times \pi}$ & 0.34 & 0.19 & $0.50^{\pi \pi}$ & -0.32 & \\
\hline NSP & & & & & & & 1 & -0.10 & $0.47^{*}$ & $0.40^{*}$ & -0.04 & 0.17 & $-0.48^{*}$ & \\
\hline PL & & & & & & & & 1 & 0.11 & -0.04 & 0.05 & 0.11 & $0.64^{\pi \times \pi}$ & \\
\hline GY & & & & & & & & & 1 & 0.27 & $0.51^{*}$ & $0.78^{* \star *}$ & -0.11 & \\
\hline BY & & & & & & & & & & 1 & $-0.47^{\star}$ & -0.37 & -0.23 & \\
\hline PHI & & & & & & & & & & & 1 & $0.81^{* \star \star}$ & 0.19 & \\
\hline HI & & & & & & & & & & & & 1 & 0.07 & \\
\hline HSW & & & & & & & & & & & & & 1 & \\
\hline
\end{tabular}

\section{Analysis of Residual Soils}

Soil samples collected after the harvest of common bean genotypes were analyzed for different soil properties including $\mathrm{pH}$, organic matter, cation exchange capacity (CEC), electrical conductivity, exchangeable cations ( $\mathrm{Ca}, \mathrm{Mg}$ and $\mathrm{K}$ ). In addition, a few acidic soil properties such as exchangeable acidity, acid saturation, effective cation exchange capacity and Al concentration were analyzed to estimate the changes that may have occurred upon liming and growing common bean genotypes without liming. The results showed that application of lime caused an increase in $\mathrm{pH}$. The mean magnitude of increase was 0.69 units (Table 1 ), but growing common bean genotypes on the lime untreated acid soil further decreased the $\mathrm{pH}$ of the soil by 0.06 units in this experiment. In general, the result obtained for the soil analysis after crop harvest indicted very strong acid soil $\left(\mathrm{pH}_{\mathrm{H} 2 \mathrm{O}}, 4.48\right)$, low exchangeable cations and their fractions (Table 1). On the other hand, growing common bean genotypes without lime on strong acid soil resulted in an increase in acid saturation. Lime application resulted in decreased acid saturation by half (Table 1). The concentration of available $P$ was very low under lime untreated and low when lime was applied after the crop harvest (Table 1).

\section{DISCUSSION}

The bean genotypes responded markedly to soil acidity in which case distinct symptoms of chlorosis of lower leaves as well as absence of root nodules were observed. The chlorosis, which may have been attributable to lack of nitrogen fixation as suggested by Piha and Munns (1987), was distinctly visible especially at early vegetative stage of growth. For a few genotypes, very few and tiny nodules where observed, which were difficult to separate from root and soil. Generally, the genotypes failed to produce nodules at Nedjo site when grown on both lime treated and untreated acid soils. Consistent with the result of his study, Evans et al. (1980) reported that nodulation was 10 times more sensitive to acidity than the growth of rhizobium or the legume root alone. In addition similar to the findings of this study, Vargas and Graham (1989) found that soil acidity is a major factor limiting nodulation and nitrogen fixation in common bean. Soil acidity adversely affects nodulation and nitrogen fixation was also reported by Bambara and Ndakidemi (2010) who observed that drastically affected legume-rhizobium symbiosis.

Growing common bean on acid soils significantly affect phenology and growth of the genotypes. 
Hirpa Legesse et al.,

However, lime application reduces the toxicity effects of soil acidity and improves the growth performance of the genotypes. In general the genotypes reached physiological maturity significantly earlier when lime was applied than when lime was not applied. Similar results also were reported by Meda and Furlani (2005), the presence of $\mathrm{Al}$ in nutrient solution caused a delay in the vegetative growth of the tropical leguminous plants used as cover crops in Brazil. Taller plants were observed in response to growing the genotypes under lime treated soil than under untreated soil. Corroborating the results of this study, Oluwatoyinbo et al. (2005) reported that plant height was significantly increased by the application of lime. This may be attributed to the toxic effect of soil acidity, which may lead to stunting of plants under lime untreated soil. This suggestion is consistent with the results of Zhang et al. (2007) who reported that soil acidity led to Al-induced leaf necrosis. The result of this study is consistent also with that of Foy (1984), who reported leaf yellowing in response to low soil $\mathrm{pH}$ and also with that of Wang et al. (2006) who stated that soil acidity led to stunted leaf growth. The result of this study agrees also with that of Rout et al. (2001) who reported that low soil pH led to late leaf maturity as a result of Al-toxicity.

Considerable variability for soil acidity tolerance among the bred lines and improved genotypes has been observed in this study. Similar results were reported by Rao et al. (2004) for acid soil adaptation and Rangel et al. (2005) for Al resistance among common bean genotypes. Large genotypic differences among crops also have been reported for Al tolerance by Foy et al. (1972) and Noble et al., (1985). Similarly, consistent with the results of this study, differing tolerance to aluminium tolerance of genotypes of Phaseolus vulgaris, Glycine max, Zea mays and Avena sativa were reported earlier by Foy (1988).

The results obtained in this study also revealed that some genotypes, namely, New BILFA 58, SER 176 and Beshbesh, produced significantly higher absolute as well as relative grain yields. On the other hand, only a few genotypes, namely, Dimitu, new BILFA 51, and new BILFA 61, did produce lower absolute yield whilst producing higher relative yield. Therefore, relative yield data should not be used alone as a selection criterion for tolerance to soil acidity. Consistent with this result, Fisher and Scott (1987) showed that equally responsive genotypes may have equal absolute tolerances yield differential between blocks but appear quite different in their yield ratios. Thus, the ratios should be interpreted with caution, or at least not reported alone.

\section{Sci. Technol. Arts Res. J., July-Sep 2013, 2(3): 03-15}

Days to maturity had a stong and negtive relation with number of pods per plant, grain yield, pod harvest index and harvest index in soil not treated with lime. It also had a weak and negtive correlation with grain yield of genotypes grown in soil treated with lime ( Table 7). Similar results were also reported by Salehi et al. (2008), Assady et al. (2005), and Kumar et al. (2002) for common bean. As observed from the results, genotypes that produced higher grain yields under both lime untreated and lime- treated soil were early in maturity.

Number of pods per plant was positively correlated with plant height in both unlimed and limed soils. However, the correlation between plant height and pod length was weak and negative in unlimed soil. In line with this result, Arya et al. (1999), reported that the number of pods per plant was positively correlated with plant height. In both lime untreated and treated soils 100 seed weight was strongly and positively correlated with pod length and weakly correlated with the number of pods in unlimed soil. However, 100 seed weight was weakly and negatively correlated with number of seeds per pod in both unlimed and limed soil. Similarly, Changezi et al. (2005) and Dursum (2007) reported the highest positive correlation with seed yield for the number of pods per plant and 100-seed weight. These results give a clear indication that the grain yield was mutually very closely associated with number of pods per plant, in both unlimed and limed soil. It seems that this is useful characters to select for high yield in common bean breeding programs for soil acidity tolerance.

\section{CONCLUSIONS}

Soil acidity problems for common bean production can be overcome by growing genotypes which are adapted to acid soil condition in circumstances where other soil amendment strategies are not readily practical. However, this is not possible until these tolerant genotypes are developed. This study revealed that common bean genotypes differ in tolerance to soil acidity. Although some genotypes exhibited an outstanding performance in terms grain yield and yield related traits, soil fertility improvement through liming would still be very important if economical bean production is to be produced in places with strong acid soil as the one used in this study. Moreover, the great variability of 25 common bean genotypes exhibited a good potential to screening large germplasm of common bean for soil acidity tolerance and develop a cultivar that are tolerant to soil acidity with potential and quality grain on such acid soils in the future. 
Hirpa Legesse et al.,

\section{ACKNOWLEDGMENTS}

We are grateful to the financial grant of the Ministry of Education through the Haramaya University. We would like to thank Melkassa Agricultural Research Centre for facilitating the research work and also acknowledge the staff members of Nedjo TVET College for their technical assistance during the time of conducting the field experiment.

\section{REFERENCES}

Abdenna Deressa, Negassa Chewaka and Tilahun Geleto.( 2007). Inventory of Soil Acidity Statusin Crop Lands of Central and Western Ethiopia. (http://www.tropentag.de/2007/abstracts/links/De ressa_kmwmgGas.pdf).

Arya, P.S., Ajai, R. and Rana, A. (1999). Study of direct and indirect influence of some yield traits on green pod yield in French bean (Phaseolus vulgaris L.). Advances in Horticulture and Floriculture 6:99-106.

Assady, B., Dorri, H.R. and Vaezi, S. (2005). Study of genetic diversity of bean (Phaseolus vulgaris L.) genotypes by multivariate analysis methods. The first Iranian Pluses symposium, research centre for plant sciences. Ferdowsi University of Mashhad, Mashhad, Iran. Pp.650.

Bambara, S. and Ndakidemi, PA. (2010). The potential roles of lime and molybdenum on the growth, nitrogen fixation and assimilation of metabolites in nodulated legume: A special referenceto Phaseolus vulgaris L. African Journal of Biotechnology 8(17):2482-2489.

Bray, R.H. and Kurtz, LT. (1945). Determination of total organic and available phosphorus in soils. Soil Science 59:39-45.

Changezi, M.S., Khaghani and Khaghani, S. (2005). Trait correlations and yield component analysis of local beans in Arak. The first Iranian pulse symposium, research centre for plant sciences, Ferodwsi University of Mashad, Iran.Pp.321.

CSA (Central Statistical Authority). (2012). Crop production Forecast sample survey. Report on area and crop production forecast for major grain crops. Bulletin Statistics, Vol. I, Addis Ababa, Ethiopia.

Dursun, A. (2007). Variability, heritability and correlation studies in bean (Phaseolus vulagris L.) genotypes. World Journal of Agricultural Sciences 3(1): 12-16.

Eswaran, H., Reich, P. and Beinroth, F. (1997). Global distribution of soils with acidity. In: PlantSoil Interactions at Low pH. Moniz, A.C (eds.). Brazilian Soil Science Society. Pp. 159-164.
Sci. Technol. Arts Res. J., July-Sep 2013, 2(3): 03-15

Evans, L. S., Lewin, K.F. and Vella, FA. (1980). Effect of nutrient $\mathrm{pH}$ on symbiotic nitrogen fixation by Rhizobium leguminosarum and Pisum sativum. Plant and Soil 56: 71-80.

Fageria, N.K., Santos, A.B. and Baligar, V.C. (1997). Phosphorous Soil Test Calibration for Lowland Rice on an Inceptisol. Agronomy Journal 89:737-742.

Fisher, J.A. and Scott, B.J. (1987). Response to selection for aluminum tolerance. In: PGE Searle, BG Davey, eds, Priorities in Soil/Plant Relations: Research for Plant Production. The University of Sydney, Sydney, Australia, pp 135137

Foy, C.D. (1988). Plant adaptation to acid, aluminum toxic soils. Commendams of Soil Science. Plant Analysis 19:959-987.

Foy, C.D., Fleming, A.L. and Gerloff, G.C. (1972). Differential aluminium tolerance in two snap bean varieties. Agronomy Journal 64:815-818.

Foy, C.D. (1984). Physiological effects of hydrogen, aluminum, and manganese toxicities in acid soil. In: Soil Acidity and Liming. Adams, F. (ed.). American Society of Agronomy, Inc., Madison, WI. pp. 57-97.

Jackson, M.L. (1958). Soil Chemical Analysis. Prentice Hall Inc., Engle Wood Cliffs, NJ, USA. Subtropics. Longman Scientific and technical, Essex, New York.

Johnson, J.P., Craver, B.F. and Baligar V.C. (1997). Productivity in Great Plains acid soils of wheat genotypes selected for aluminium tolerance. Plant and Soil 188: 101-106.

Kochian, L.V., Hoekenga, O.A. and Piners, M.A. (2004). How do crop plants tolerate acid soils: Mechanism of aluminum tolerance and phosphorus efficiency. Annual Review of Plant Biology 55: 459-493.

Kumar, J., Singh, H., Singh, T., Tonk, D.S., Lal, R. (2002). Correlation and path coefficient analysis of yield and its components in summer mung (Vigna radiata L.). Crop Research 24: 374-377.

Manrique, G., Rao, I.M., Beebe, S. (2006). Identification of aluminum resistance common bean genotypes using hydroponic screening method. Paper presented at the $18^{\text {th }}$ world congress of soil sciences, Philadephia, USA, July 9-15.

McLean, E.O. (1965). Aluminium. pp. 978-998. In: C.A. Black (Ed.). Methods of Soil Analysis. Agronomy. No. 9. Part II. American Society of Agronomy, Madison, Wisconsin, USA. 


\section{Hirpa Legesse et al.,}

Meda, A.R. and Furlani, P.R. (2005). Tolerance to Aluminum Toxicity by Tropical Leguminous Plants Used as Cover Crops. Brazilian Archives of Biology and Technology 48(2):309-317.

Mesfin Abebe. (2007). Nature and Management Of Acid Soils In Ethiopia. Addis Ababa, Ethiopia.

Noble, A.D., Lea, J.D., and Fey, M.V. (1985). Genotypic tolerance of selected dry bean (Phaseolus vulgaris L.) cultivars to soluble Al and to acid, low $\mathrm{P}$ soil conditions. South African Journal of Plant Soil 2:113-119.

Oluwatoyinbo, F.I., Akande M.O. and. Adediran J.A. (2005). Response of Okra (Abelmoschus esculentus) to Lime and Phosphorus Fertilization in an Acid Soil. World Journal of Agricultural Sciences 1(2):178-183,

Piha, M. I. and Munns, D. N. (1987). Sensitivity of common bean (Phaseolus vulgaris L.) symbiosis to high soil temperatures. Plant and Soil 98:183194.

Rangel, A.F., Mobin, M., Rao, I.M. and Horst, W.J. (2005). Proton toxicity interferes with the screening of common bean (Phaseolus vulgaris L.) genotypes for aluminium resistance in nutrient solution. Journal of Plant Nutrition and Soil Science 168:607-616.

Rao, IM. (2001). Role of physiology in improving crop adaptation to abiotic stresses in the tropics: the case of common bean and tropical forages. Marcel Dekker, New York, USA, pp 583-613

Rao, I.M., Beebe, S., Ricaurte, J., Teran, H. and Singh, S. (2004). Common bean (Phaseolus vulgaris L.) genotypes tolerant to aluminium toxicity soils in the tropics. Proceedings of the $6^{\text {th }}$ International symposium on plant Soil interactions at low pH (PSILP) held in Sendai, Japan from 31 July to 5, August 2004. Japanese society of sol science and Plantnutria., Sendai, Japan, pp. 272-273.

Rout, G.R., Samantaray, S. and Das, P. (2001). Aluminium toxicity in plants:a review. Agronomie 21: 2-21.
Sci. Technol. Arts Res. J., July-Sep 2013, 2(3): 03-15

Salehi, M., Tajik, M. and Ebadi, A.G. (2008). The study of relationship between different traits in common bean (Phaseolus vulgaris L.) with multivariate statistical methods. AmericanEurasian Journal of Agricultural \& Environmental Science 3: 806-809.

SAS Institute Inc. (2004). SAS/STAT User's Guide: Version 9.1th edn. SAS Institute Inc., Cary,North Carolina.

Setegn, Gebeyehu. (2006). Physiological response to drought stress of common bean (Phaseolus vulgaris L.) genotypes differing in drought resistance. PhD Dissertation, Gottingen University, Germany.

Tesso, B. (2007). Yield and canning quality attributes of Navy beans (Phaseolus vulgaries L.) as influenced by genotype and environment(un Published MSc Thesis, 2007), Haramaya University

Thompson, M. and Banerjee, E.K. (1991). In: Haswell, S.J. (ed.) Atomic absorption spectrometery, Elsevier, Amsterdam, pp 289320.

.Vargas, A. A. T. and Graham, P. H. (1989). Cultivar and $\mathrm{pH}$ effect on competition for nodule sites between isolates of Rhizobium in beans. Plant and Soil 17: 195-200.

Walkley, A. and Black, C.A. (1934). An examination of Detergent method for determining soil organic matter and proposed modification of the proposed modification of the chromic acid titration method. Soil Science 37: 29-38

Wang, J., Raman, H. Zhang, G. Mendham, N. and Zou, M. (2006). Aluminium tolerance in barely (Horidium vulgaris L.):Physiological mechanisms, genetic and screening methods. Journal of Zhejiang University Science 7: 769-787.

Zhang, Xiao-Bin, Peng, L., Yang, Y.S. and Gen-Di xu. (2007). Effect of Al in soil on photosynthesis and related morphological and physiological characteristics of two soybean genotypes. Botanical Studies 48: 435-444. 